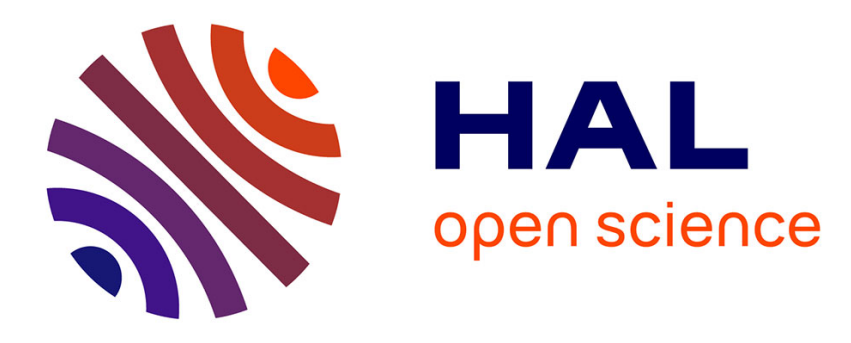

\title{
On the saturation rule for the stability of queues
}

François Baccelli, Serguei Foss

\section{To cite this version:}

François Baccelli, Serguei Foss. On the saturation rule for the stability of queues. [Research Report] RR-2015, INRIA. 1993. inria-00074656

\section{HAL Id: inria-00074656 https://hal.inria.fr/inria-00074656}

Submitted on 24 May 2006

HAL is a multi-disciplinary open access archive for the deposit and dissemination of scientific research documents, whether they are published or not. The documents may come from teaching and research institutions in France or abroad, or from public or private research centers.
L'archive ouverte pluridisciplinaire HAL, est destinée au dépôt et à la diffusion de documents scientifiques de niveau recherche, publiés ou non, émanant des établissements d'enseignement et de recherche français ou étrangers, des laboratoires publics ou privés. 


\section{On the Saturation Rule for the Stability of Queues}

François Baccelli

Scrouci Foss

$$
N^{\circ} 2015
$$

Septembre 1993

PROGRAMME 1

Architectures parallèles,

balses de donnéses,

réscilux el systèmes distribués

\section{apport}

\section{de recherche}




\title{
La règle de saturation pour la stabilité des réseaux de files d'attente
}

\author{
François Baccelli* et Serguei Foss ${ }^{\dagger}$
}

31 août 1993

\begin{abstract}
Résumé
Cet article étudie la stabilité de réseaux de files d'attente ouverts sous des hypothèses stationnaires ergodiques. On définit un ensemble de conditions, appelé hypothèse séparable-monotone, sous lesquelles la région de stabilité est donnée par la règle suivante: saturer les files d'attente qui reçoivent des clients du flux d'arrivée; soit $\mu$ l'intensité du processus des départs du réseau ainsi saturé; alors le réseau est stable si $\lambda<\mu$, instable si $\lambda>\mu$. Lorsque cette condition de stabilité cst satisfaite, on montre qu'il est possible de construire un régime stationnaire minimal pour certaines variables d'état associées au réseau. Cet ensemble d'hypothéses contient deux propriétés trajectorielles, la monotonie externe et la séparabilité, qui sont satisfaites par de nombreux types de réseaux. L'outil mathématique principal pour la preuve de cette règle est la théorie ergodique sous-additive.
\end{abstract}

Mots Clés: Réseaux de files d'attente ouverts, processus ponctuels stationnaires, séparabilité, monotonie, théorèmes ergodiques de premier ordre et de deuxième ordre, théorème sous-additif ergodique,

\footnotetext{
-INRIA Centre Sophia Antipolis 06565 Valbonne France; baccelli@sophia.inria.fr; Travaux effectués dans le cadre du projet BRA Qmips, financé par la Commission Européenne, DG XIII

INOVOSIBIRSK State University, 630090 Novosibirsk, Russie; foss@math.nsk.su; Travaux effectués dans le cadre d'une année sabbatique à l'INRIA Sophia Antipolis
} 


\title{
On the Saturation Rule for the Stability of Queues
}

\author{
François Baccelli* and Serguei Foss ${ }^{\dagger}$
}

August 31, 1993

\begin{abstract}
This paper focuses on the stability of open queueing systems under stationary ergodic assumptions. It defines a set of conditions, the monotone separable framework, ensuring that the stability region is given by the following saturation rule: 'saturate' the queues which are fed by the external arrival stream; look at the 'intensity' $\mu$ of the departure stream in this saturated system; then stability holds whenever the intensity of the arrival process, say $\lambda$ satisfies the condition $\lambda<\mu$, whereas the network is unstable if $\lambda>\mu$. Whenever the stability condition is satisfied, it is also shown that certain state variables associated with the network admit a finite stationary regime which is constructed pathwise using a Loynes type backward argument. This framework involves two main pathwise properties, external monotonicity and separability, which are satisfied by several classical queueing networks. The main tool for the proof of this rule is sub-additive ergodic theory.
\end{abstract}

Keywords: Open queueing network, stationary point processes, monotonicity, separability, first and second-order ergodic properties, subadditive ergodic theorem.

*INRIA Centre Sophia Antipolis 06565 Valbonne France; baccelli@sophia.inria.fr; the work of this author was supported in part by a grant from the European Commission DG XIII, under the BRA Qmips contract

†NOVOSIBIRSK State University, 630090 Novosibirsk, Russia; foss@math.nsk.su; the work of this author was supported by a sabbatical grant from INRIA Sophia Antipolis 


\section{Introduction}

A folk theorem of queueing theory states that the stability region of an open queueing systems can be obtained as follows: 'saturate' the queues which are fed by the external arrival stream with an infinite customer population; if $\mu$ denotes the 'intensity' of the departure stream in this saturated system, then the system is stable when the intensity of the arrival process, $\lambda$, satisfies $\lambda<\mu$. This engineering rule, which we will refer to as the saturation rule, was initially designed for Markovian queueing systems, where $\mu$ could be obtained by computing the steady state of a Markov chain of smaller dimension than that of the initial non-saturated system (see for instance [8]).

The aim of the present paper is to set a natural framework in which this rule can be rigorously proved when dropping the Markovian assumptions and replacing them by standard stationary ergodic assumptions, namely the arrival point process is a stationary ergodic marked point process of finite intensity. The conditions required by this framework consist of easy-to-check, pathwise properties of the system under consideration. The two main conditions are external monotonicity and separability, although two other mild conditions called causality and homogeneity are also assumed.

For any queueing system within this framework (to be defined below), we will denote $X_{n}$ the time of the last activity to take place in the system, whenever one starts with $n$ customers, all arrived at time 0 in an empty system. This notion of time to inactivity, turns out to be the adequate way of implementing the saturation idea for non-Markov systems. The main result is then:

Theorem 1 The sequence $\left\{X_{n}\right\}$ satisfies a SLLN:

$$
\lim _{n} \frac{X_{n}}{n}=\gamma(0) \text { a.s., }
$$

for some non-negative constant $\gamma(0)$; this constant will also be finite if the input marked process satisfies natural integrability conditions. Whenever the intensity of the input process satisfies the condition $\lambda<\gamma^{-1}(0)$, then the system is stable, whereas it is unstable if $\lambda>\gamma^{-1}(0)$. 
The stability here means that the time to inactivity (usually the time to empty the system) when it is fed by the restriction of the point process on $(-\infty, t)$ admits a finite steady state regime. This usually implies most standard acceptations of stability. This stationary regime is constructed pathwise using a backward argument of the same nature as the argument of Loynes in the $G / G / 1$ queue. If $\lambda>\gamma^{-1}$, the system is unstable in the sense that the time to inactivity tends to $\infty$ in probability.

Several classes of queueing systems for which the stability is an open question (at least in the stationary ergodic case) fall within this framework, like for instance Jackson type queueing networks. It also contains other classes of discrete event systems like certain classes of Petri nets. Various examples and counter-examples are discussed in the final section.

\section{The Monotone Separable Framework}

Arrival Point Process Let $N$ be a marked point process with points $\left\{T_{n}\right\}_{n \in \mathbb{Z}}$ and marks $\left\{\xi_{n}\right\}_{n \in \mathbb{Z}}$, where $\xi_{n} \in(K, \mathcal{K})$. This point process is not assumed to be simple (nor to be stationary at this stage). We only assume that $T_{n} \leq T_{n+1}$, for all $n$. We shall use the notations $\tau_{n}$ for $T_{n+1}-T_{n}, c+N$ for the point process $\left\{T_{n}+c\right\}$ and $c N$ for the point process $\left\{c T_{n}\right\}$, where $c \in I R$. In what follows, we shall not adopt the usual renumbering rule for point processes, and the $n$-th point of $N+c$ will be $T_{n}+c$ by definition.

First Order State Variables For all $m \leq n \in I N$, let $X_{[m, n]}(N)$ be the time of the last activity in the network, when this one starts empty and is fed by the $[m, n]$ restriction of $N$, namely the point process $\left\{T_{l}\right\}_{m \leq l \leq n}$. We assume to be given a set of functions $\left\{f_{l}\right\}, f_{l}: I R^{l} \times K^{l} \rightarrow I R$, such that:

$$
X_{[m, n]}(N)=f_{n-m+1}\left\{\left(T_{l}, \xi_{l}\right), m \leq l \leq n\right\},
$$

for all $n, m$ and $N$. We assume that the functions $f_{n}$ are such that the following properties hold for all $N$ :

1. (causality): For all $m \leq n$,

$$
X_{[m, n]}(N) \geq T_{n}
$$


2. (external monotonicity): For all $m \leq n$,

$$
X_{[m, n]}\left(N^{\prime}\right) \geq X_{[m, n]}(N)
$$

whenever $N^{\prime} \stackrel{\text { def }}{=}\left\{T_{n}^{\prime}\right\}$ is such that $T_{n}^{\prime} \geq T_{n}$ for all $n$;

3. (homogeneity): $\forall c \in I R, \forall m \leq n$

$$
X_{[m, n]}(c+N)=X_{[m, n]}(N)+c
$$

4. (separability): If, for all $m \leq l<n, X_{[m, l]}(N) \leq T_{l+1}$, then

$$
X_{[m, n]}(N)=X_{[l+1, n]}(N) \text {. }
$$

In words, property (4) simply states that if the arrival of customer $l+1$ takes place later than the last activity for the arrival process $[m, l]$, then the evolution of the network after time $T_{l+1}$ is the same as in the network which 'starts empty' at this time.

Properties of the State Variables Let

$$
Z_{[m, n]}(N) \stackrel{\text { def }}{=} X_{[m, n]}(N)-T_{n}=X_{[m, n]}\left(N-T_{n}\right) .
$$

Note that $Z_{[m, n]}(N)$ is a function of $\left\{\xi_{n}\right\}$ and $\left\{\tau_{l}\right\}_{m \leq l \leq n-1}$ only. In particular, $Z_{n}(N) \stackrel{\text { def }}{=} Z_{[n, n]}(N)$ is not a function of $\left\{\tau_{n}\right\}$.

Lemma 1 (internal monotonicity of $X$ and $Z$ ) Under the above conditions, the variables $X_{[m, n]}$ and $Z_{[m, n]}$ satisfy the internal monotonicity property: for all $N$

$$
X_{[m-1, n]}(N) \geq X_{[m, n]}(N), \quad Z_{[m-1, n]}(N) \geq Z_{[m, n]}(N) \quad(m \leq n) .
$$

Proof Consider the point process $N^{\prime}$ with points:

$$
T_{j}^{\prime}= \begin{cases}T_{j}-Z_{m-1}(N) & \text { for } j \leq m-1 \\ T_{j} & \text { for } j \geq m .\end{cases}
$$

Since the $[m, \infty]$ restrictions of $N$ and $N^{\prime}$ coincide, $X_{[m, n]}(N)=X_{[m, n]}\left(N^{\prime}\right)$. The separability assumption implies that $X_{[m-1, n]}\left(N^{\prime}\right)=X_{[m, n]}\left(N^{\prime}\right)$. Finally, the external monotonicity implies that $X_{[m-1, n]}\left(N^{\prime}\right) \leq X_{[m-1, n]}(N)$. 
Lemma 2 (subadditive property of $Z$.) Under the above conditions, $\left\{Z_{[m, n]}\right\}$ satisfies the following sub-additive property: for all $m \leq l<n$, for all $N$

$$
Z_{[m, n]}(N) \leq Z_{[m, l]}(N)+Z_{[l+1, n]}(N)
$$

Proof Introduce two auxiliary point processes $N^{1}=\left\{T_{j}^{1}\right\}$ and $N^{2}=\left\{T_{j}^{2}\right\}$ defined by

$$
T_{j}^{1}= \begin{cases}T_{j} & \text { for } j \leq l \\ T_{j}+Z_{[m, l]}(N) & \text { for } j>l\end{cases}
$$

and

$$
T_{j}^{2}= \begin{cases}T_{j}-Z_{[m, l]}(N) & \text { for } j \leq l \\ T_{j} & \text { for } j>l\end{cases}
$$

So $T_{j}^{2}=T_{j}^{1}-Z_{[m, l]}(N)$, for all $j$. Then, using assumptions (1)-(4) of our framework

$$
\begin{aligned}
& X_{[m, n]}(N) \stackrel{2}{\leq} X_{[m, n]}\left(N^{1}\right) \stackrel{4}{=} X_{[l+1, n]}\left(N^{1}\right) \\
& \stackrel{3}{=} X_{[l+1, n]}\left(N^{2}\right)+Z_{[m, l]}(N)=X_{[l+1, n]}(N)+Z_{[m, l]}(N) \text {. }
\end{aligned}
$$

Therefore

$$
\begin{aligned}
Z_{[m, n]}(N) & =X_{[m, n]}(N)-T_{n} \leq X_{[l+1, n]}(N)-T_{n}+Z_{[m, l]}(N) \\
& =Z_{[l+1, n]}(N)+Z_{[m, l]}(N) .
\end{aligned}
$$

\section{Proof of the Saturation Principle}

Stationarity Assumptions Assume the variables $\left\{\tau_{n}, \xi_{n}\right\}$ are random variables defined on a common probability space $\left(\Omega, \mathcal{F}, P^{0}, \theta\right)$, where $\theta$ is measure-preserving shift transformation, such that $\left(\tau_{n}, \xi_{n}\right) \circ \theta=\left(\tau_{n+1}, \xi_{n+1}\right)$. For instance, this space is the Palm space of a stationary ergodic point process. The following integrability assumptions are also assumed to hold:

$$
E^{0} \tau_{n} \stackrel{\text { def }}{=} \lambda^{-1}<\infty, \quad E^{0} Z_{n}<\infty
$$


First-Order Ergodic Property Kingman's sub-additive ergodic theorem gives:

Lemma 3 There exists a finite non-negative constant $\gamma$ such that the a.s. limits

$$
\lim \frac{Z_{[-n,-1]}}{n}=\lim \frac{E^{0} Z_{[-n,-1]}}{n}=\lim \frac{Z_{[1, n]}}{n}=\lim \frac{E^{0} Z_{[1, n]}}{n}=\gamma
$$

hold $P^{0}$-a.s.

Corollary 1 Under the foregoing assumptions

$$
\lim _{n} \frac{X_{[1, n]}}{n}=\gamma+\lambda^{-1}
$$

0-1 Law Let $A$ be the event $A=\left\{\lim Z_{[-n, 0]}=\infty\right\}$.

Theorem 2 Under the foregoing ergodic assumption, $P^{0}(A) \in\{0,1\}$.

Proof Note that $\theta A=\left\{\lim Z_{[-n,-1]}=\infty\right\}$. But owing to the sub-additive property, $Z_{[-n,-1]} \geq Z_{[-n, 0]}-Z_{0}$. This and the integrability of $Z_{0}$ imply that $\theta A \supseteq A$. Since $\theta$ is ergodic, the proof is concluded.

Scaling Factor For all $0 \leq c<\infty$, the sequences

$$
X_{[m, n]}(c N) \stackrel{\text { def }}{=} f_{n+1-m}\left\{\left(c \cdot T_{l}, \xi_{l}\right) ; m \leq l \leq n\right\}
$$

and

$$
Z_{[m, n]}(c N)=X_{[m, n]}(c N)-c \cdot T_{n}
$$

satisfy all the monotonicity and sub-additive properties mentioned above. In addition, for all $n$

(a) $Z_{[-n,-1]}(c N)$ is decreasing in $c$; Thus

(b) $X_{[1, n]}(c N)$ is increasing in $c$.

Lemma 4 For all $c \geq 0$, there exists a non-negative constant $\gamma(c)$ such that

$$
\lim \frac{Z_{[-n,-1]}(c N)}{n}=\gamma(c) \quad \text { a.s.; }
$$

$\gamma(c)$ is decreasing in $c$ while $\gamma(c)+c \lambda^{-1}$ is increasing in $c$. 
Second-Order Ergodic Property The main result on the stability region is:

Theorem 3 If $\lim Z_{[-n, 0]}(N)=\infty$ a.s., then $\lambda \gamma(0) \geq 1$. If $\lambda \gamma(0)>1$, then $\lim Z_{[-n, 0]}=\infty$ a.s.

Proof We first prove the second assertion. Let $Q$ be the point process with all its points equal to $0: T_{n}(Q)=0$ for all $n$. For $n$ fixed, let $N^{n}$ be the point process with points $T_{j}^{n}=T_{-n}-T_{0}$, for all $j$. Then

$$
\begin{aligned}
Z_{[-n, 0]}(N) & =X_{[-n, 0]}(N)-T_{0}{ }^{2} X_{[-n, 0]}\left(N^{n}\right) \\
& \stackrel{3}{=} X_{[-n, 0]}(Q)+T_{-n}-T_{0}=Z_{[-n, 0]}(Q)+T_{-n}-T_{0}
\end{aligned}
$$

and

$$
\liminf \frac{Z_{[-n, 0]}(N)}{n} \geq \gamma(0)-\lambda^{-1}>0,
$$

which concludes the proof of the second assertion.

We now prove the first one. For each integer $l \geq 1$, let $K_{l}$ be the random variable

$$
K_{l}=\min \left\{n \geq 1: \quad Z_{[-n, 0]}(N) \geq T_{l}-T_{0}\right\},
$$

which will be $P^{0}$ a.s. finite if $Z_{[-n, 0]}$ tends to $\infty$. Owing to the sub-additive property, for all $n, l \geq 1$

$$
Z_{[-n, l]} \leq Z_{[-n, 0]}+Z_{[1, l]} \leq Z_{[-n, 0]}+\sum_{i=1}^{l} Z_{i}
$$

where the random variables $Z_{i}=Z_{0} \circ \theta^{i}$ do not depend on the inter-arrival times and are integrable. For all $n \geq 1$, let $\widehat{N}^{n}$ be the point process with points

$$
\hat{T}_{j}^{n}= \begin{cases}T_{j}-T_{0} & \text { for } j \leq 0 \\ Z_{[-n, 0]}(N) & \text { for } j \geq 1\end{cases}
$$

and let $\widetilde{N}^{n}$ be defined by

$$
\tilde{T}_{j}^{n}=Z_{[-n, 0]}, \quad \text { for all } j .
$$


Then

$$
\begin{aligned}
\left(X_{[-n, l]}(N)-J_{0}\right) 1_{n \geq K_{l}} & \stackrel{2}{\leq} X_{[-n, l]}\left(\widehat{N}^{n}\right) 1_{n \geq K_{l}} \\
& \stackrel{4}{=} X_{[1, l]}\left(\widehat{N}^{n}\right) 1_{n \geq K_{\iota}}=X_{[1, l]}\left(\widetilde{N}^{n}\right) 1_{n \geq K_{\iota}} \\
& \stackrel{3}{=}\left(Z_{[-n, 0]}(N)+X_{[1, l]}(Q)\right) 1_{n \geq K_{l}} \\
& =\left(Z_{[-n, 0]}(N)+Z_{[1, l]}(Q)\right) 1_{n \geq K_{l}} .
\end{aligned}
$$

Therefore

$$
\begin{aligned}
Z_{[-n, l]}(N) 1_{n \geq K_{l}} & =\left(X_{[-n, l]}(N)-T_{l}\right) 1_{n \geq K_{l}} \\
& \leq\left(Z_{[-n, 0]}(N)+Z_{[1, l]}(Q)-T_{l}+T_{0}\right) 1_{n \geq K_{l}}
\end{aligned}
$$

Finally,

$$
\begin{aligned}
& Z_{[-n, l]}(N)-Z_{[-n, 0]}(N) \\
& \quad=\left(Z_{[-n, l]}(N)-Z_{[-n, 0]}(N)\right)\left[1_{n<K_{l}}+1_{\left.n \geq K_{l}\right]}\right. \\
& \quad \leq\left(\sum_{i=1}^{l} Z_{i}\right) 1_{n<K_{l}}+\left(Z_{[1, l]}(Q)-T_{l}+T_{0}\right) 1_{n \geq K_{l}} \\
& \quad=\Psi_{l} 1_{n<K_{l}}+Z_{[1, l]}(Q)-T_{l}+T_{0},
\end{aligned}
$$

where

$$
\psi_{l} \stackrel{\text { def }}{=} \sum_{i=1}^{l} Z_{i}-Z_{[1, l]}(Q)+T_{l}-T_{0}
$$

is $P^{0}$-integrable. By making use of the relations $Z_{[-n, l]}=Z_{[-n-l, 0]} \circ \theta^{l}$, $Z_{[-n-l, 0]} \geq Z_{[-n, 0]}$ and $E^{0} Z_{[-n-l, 0]}<\infty$, we obtain from $(2)$ that

$$
0 \leq E^{0} Z_{[-n, l]}-E^{0} Z_{[-n, 0]} \leq E^{0}\left\{\psi_{l} 1_{n<K_{l}}\right\}+E^{0} Z_{[1, l]}(Q)-l \lambda^{-1}
$$

If $K_{l}$ is a.s. finite for all $l$, the right-hand side of the last equation tends to $E^{0} Z_{[1, l]}(Q)-l \lambda^{-1}$ as $n \rightarrow \infty$. Therefore

$$
\frac{E^{0} Z_{[1, l]}(Q)}{l} \geq \lambda^{-1}
$$

for all $l$. Finally, when letting $l$ go to infinity and when making use of Lemma 4 , we obtain

$$
\gamma(0)=\lim _{l} \frac{E^{0} Z_{[1, l]}(Q)}{l} \geq \lambda^{-1}
$$


Thus if $\lambda \gamma(0)<1$, the random variable

$$
Z \stackrel{\text { def }}{=} \lim _{n} Z_{[-n, 0]} \quad \text { a.s. }
$$

is $P^{0}$-a.s. finite and it provides a minimal stationary regime for the time to inactivity, which is defined as the time to the last activity in the system when subject to the $[-\infty, 0]$ restriction of $N$.

\section{Examples and Counter-Examples}

The $G / G / 1$ Queue. Here $X_{[m, n]}$ is the departure epoch of customer $n$, when there are $n+1-m$ customers with arrival epochs $T_{l}$ and service times $\sigma_{l}$, $m \leq l \leq n ; Z_{[m, n]}$ is then the sojourn time of customer $n$. The computation of $\gamma(0)$ is trivial, by the strong law of large numbers.

The $G / G / s$ Queue. $X_{[m, n]}$ is the last departure time from the queue with customers arriving at $T_{l}, m \leq l \leq n$, that is

$$
Z_{[m, n]}(N)=\max \left(W_{[m, n]}^{1}(N)+\sigma_{n}, W_{[m, n]}^{s}(N)\right),
$$

where $W_{[m, n]}(N)=\left(W_{[m, n]}^{1}(N), \ldots, W_{[m, n]}^{s}(N)\right)$ is the ordered workload vector at time $T_{n}-$, for this arrival process (we assume that the queue is initially empty). We have

$$
\lim _{n} \frac{Z_{[1, n]}(Q)}{n}=\gamma(0) \text { a.s., }
$$

as a consequence of Lemma 4. The computation of the constant $\gamma(0)$ is immediate from the relation

$$
\lim \frac{W_{[m, n]}^{j}(Q)}{n}=\gamma(0) \quad \text { a.s. } \quad(1 \leq j \leq s) .
$$

Indeed since

$$
\sum_{i=1}^{n} \sigma_{i}=\sum_{j=1}^{s} W_{[1, n]}^{j}(Q)
$$

the relation $\gamma(0)=E^{0}(\sigma) / s$ follows by an immediate limiting argument. 
Proof of (3) The property for $j=s$ follows from the relation $Z_{[1, n]}(Q)=$ $W_{[1, n+1]}^{s}(Q)$. In order to prove the property for all $j$, it is cnough to show that

$$
\Delta_{n} \stackrel{\text { def }}{=} W_{[m, n]}^{s}(Q)-W_{[m, n]}^{1}(Q)
$$

is such that $\Delta_{n} / n$ tends to 0 a.s. Let

$$
u_{n} \stackrel{\text { def }}{=} \max \left(\sigma_{n}, \ldots, \sigma_{n+s-1}\right), \quad \text { and } \quad U_{n} \stackrel{\text { def }}{=} \min \left(\sigma_{n}, \ldots, \sigma_{n+s-1}\right) .
$$

By comparing the original queue and the queue with workload

$$
\left(W_{[1, n]}^{1}(Q), \ldots, W_{[1, n]}^{1}(Q)\right)
$$

at time $T_{n}$ - and with constant service time $u_{n}$ over the interval $n, n+$ $1, \ldots, n+s-1$, we see that

$$
W_{[1, n+s]}^{1}(Q) \geq W_{[1, n]}^{1}(Q)+u_{n}, \quad(n \geq 0) .
$$

Similarly, when considering the queue with workload

$$
\left(W_{[1, n]}^{1}(Q), W_{[1, n]}^{s}(Q), \ldots, W_{[1, n]}^{s}(Q)\right)
$$

at time $T_{n}-$ and with constant service time $U_{n}$, we obtain

$$
W_{[1, n+s]}^{s}(Q) \leq \max \left(W_{[1, n]}^{s}(Q), W_{[1, n]}^{1}(Q)+s U_{n}\right) \quad(n \geq 0) .
$$

Thus

$$
\Delta_{n+s} \leq \max \left(s U_{n}, \Delta_{n}-u_{n}\right), \quad(n \geq 0) .
$$

The solution $\left\{D_{k}^{l}\right\}$ of the equation

$$
D_{k+1}^{l}=\max \left(s U_{k s+l}, D_{k}^{l}-u_{k s+l}\right), \quad(n \geq 0,0 \leq l<s),
$$

with initial condition $D_{0}^{l}=\Delta_{l}$, is such that $\Delta_{k s+l} \leq D_{k}^{l}$ for all $k$ and $l$. Posing $C_{k}^{l}=D_{k}^{l}-s U_{(k-1) s+l}$, we see that $\left\{C_{k}\right\}$ satisfies the Lindley equation

$$
C_{k+1}^{l}=\left(C_{k}^{l}+s U_{(k-1) s+l}-s U_{k s+l}-u_{k s+l}\right)^{+},
$$

and so $C_{k}^{l} / k$ tends to 0 a.s. for all $l$. 
FIFO Kelly-type Networks Customers are given a route through the network upon arrival (namely the route of customer $n$ is a mark associated with point $T_{n}$ ). Then the external monotonicity property is not satisfied. Note that the conditions stating that the traffic intensity be less than one at each queue is not sufficient for monotonicity, even in the FIFO case (see [6]).

Kelly-type Networks with Synchronization Constraints These networks were defined in [2]. The routing is mechanism is the same as above, but the service discipline is such that, on a given queue, service requirements brought by the $n$-th external arrival can in no case be served before all the service requirements brought by the $n-1$-st external arrival have been completed. There, $X_{[m, n]}$ is also the last departure epoch from the system with restricted arrivals as above. The constant $\gamma(0)$ is the $(\max ,+)$ Lyapunov exponent of a sequence of stationary random matrices defined from the routing and the service times (see [1] for the notions pertaining to $(\max ,+)$-Lyapunov exponents).

Jackson-type Networks Whenever the routes and the service times are stationary ergodic sequences associated with stations (namely, the $n$-th customer to reach station $j$ requires the service time $\sigma_{n}^{j}$, and is routed to station $q_{n}^{j}$, where $\left\{\sigma_{n}^{j}\right\}_{n}$ and $\left\{q_{n}^{j}\right\}_{n}$ are stationary ergodic sequences, then the network falls in the monotone separable framework. The stability region is given by the condition $\rho_{j}<1$, for all queues, where $\rho_{j}$ is the traffic intensity at queue $j$ (see [3]).

Stochastic Petri Nets Open FIFO stochastic event graphs fall in our framework, namely they satisfy the four conditions of the framework (see [1]). The constant $\gamma(0)$ is also the $(\max ,+)$ Lyapunov exponent of a sequence of random matrices defined from the topology of the network and the service times.

For general open stochastic Petri nets with switching, also defined in [1], take $X_{[m, n]}$ to be the epoch of the last firing time of whole net for the $[m, n]$ restriction of the arrival point process. Conditions 1-3 of the framework are always satisfied. The validity of condition 4 of the framework is discussed in $[4]$. 
A Non-separable Network for which the Principle Does Not Hold Consider an assembly queue with two independent Poisson arrival streams with the same intensity $\lambda / 2$. The system starts empty. Whenever there are customers of both classes in the queue, service is provided at rate $\mu$. The completion of a service consumes one customer of each class. Whenever the queue has no customers of cither class, it is blocked. Let consider as input stream the superposition of the two Poisson processes properly marked. If one saturates the system with an infinite customer population, the (Markov) departure rate is $\mu$. Similarly, if one takes the viewpoint of letting $n$ customers of this input stream arrive at time 0 , the last activity of the system takes place at time $\mu^{-1} n / 2+o(n)$. A naive application of the saturation rule would suggest that if $\lambda<\mu$, the system is stable. However, such queues are always unstable (see [1]), whatever the values of $\lambda$ and $\mu$. Note that this system does not satisfy the separability property.

\section{References}

[1] F. Baccelli, G. Cohen, G. Olsder and J.P. Quadrat. Linearity and synchronization, Wiley, 1992.

[2] F. Baccelli and Z. Liu. On a class of stochastic evolution equation s. Ann. Appl. Prob. 20-2: 350-374, 1992.

[3] F. Baccelli and S. Foss. Stability of Jackson-type queueing networks. INRIA Report No. 1945, June 1993, QUESTA, to appear.

[4] F. Baccelli and S. Foss. Stability of stochastic Petri nets with general topology. In preparation.

[5] A.A. Borovkov. Asymptotic methods in queueing theory. Wiley, 1984.

[6] M. Bramson. Instability of FIFO Queueing Networks. Preprint Mathematics Department, University of Wisconsin, 1993.

[7] J.F.C. Kingman. Subadditive ergodic theory. Annals of Proba. 1, 883$909,1973$. 
[8] S. Lavenberg, Maximum Departure Rate of certain Open Queueing Networks Having Finite Capacity Constraints, RAIRO série bleue, 1978, 12-4, Paris 
Unité de Recherche INRIA Sophia Antipolis 2004, route des Lucioles - B.P. 93 - 06902 SOPHIA ANTIPOLIS Cedex (France)

Unité de Recherche INRIA Lorraine Technopôle de Nancy-Brabois - Campus Scientifique 615, rue du Jardin Botanique - B.P. 101 - 54602 VILLERS LES NANCY Cedex (France) Unité de Recherche INRIA Rennes IRISA, Campus Universitaire de Beaulicu 35042 RENNES Cedex (France) Unité de Recherche INRIA Rhône-Alpes 46, avenue Félix Viallet - 38031 GRENOBLE Cedex (France) Unité de Recherche INRIA Rocquencourt Domaine de Voluceau - Rocquencourt - B.P. 105 - 78153 LE CHESNAY Cedex (France)

\section{EDITEUR}

INRIA - Domaine de Voluceau - Rocquencourt - B.P. 105 - 78153 LE CHESNAY Cedex (France)

ISSN $0249-6399$ 